\title{
Designing Games for the Rehabilitation of Functional Vision for Children with Cerebral Visual Impairment
}

\author{
Conor Linehan \\ Lincoln Social Computing \\ Research Centre, \\ University of Lincoln, \\ Lincoln, LN67TS, UK \\ clinehan@lincoln.ac.uk \\ Jonathan Waddington \\ WESC Foundation \\ Topsham Road, Exeter \\ Devon, EX2 6HA \\ \{Jwaddington\} \\ @wescfoundation.ac.uk
}

\author{
Timothy L. Hodgson \\ School of Psychology \\ University of Lincoln \\ Lincoln, LN67TS, UK \\ thodgson@lincoln.ac.uk \\ Kieran Hicks, Robert Banks \\ School of Computer Science, \\ University of Lincoln, \\ Lincoln, LN67TS, UK \\ clinehan@lincoln.ac.uk
}

Note: This is the author's version of the work. It is posted here by permission of ACM for your personal use. Not for redistribution. The definitive version was published in Proceedings of the 32nd international conference on Human factors in computing systems (CHI 2014) extended abstracts.

ACM 978-1-4503-2474-8/14/04

http://dx.doi.org/10.1145/2559206.2581219

\begin{abstract}
Evidence has accumulated that visual rehabilitation for patients with neurological visual impairment can be effective. Unfortunately, the existing therapy tools are repetitive, uninteresting, and unsuitable for use with children. This project aims to improve the engaging qualities of visual rehabilitation for children, through the design of therapy tools based on game design principles. Development is ongoing in a participatory, user-centred manner in conjunction with a specialist centre for childhood visual impairment. This paper outlines design requirements and briefly reports early findings of the development process.
\end{abstract}

\section{Author Keywords}

Game; Rehabilitation; Therapy; Visual Impairment;

\section{ACM Classification Keywords}

H.5.m. Information interfaces and presentation (e.g., $\mathrm{HCI}$ ): Miscellaneous.

\section{Introduction}

Recent years have seen some success in the use of games technology to improve the engaging qualities of therapy programmes (see [4][6]). For example, researchers have investigated the usefulness of commercial games consoles such as the Nintendo Wii 


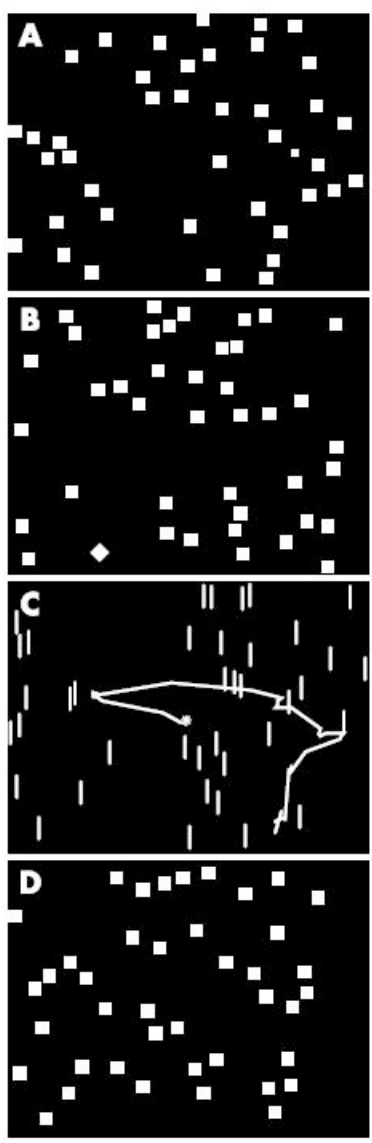

Figure 1. Four examples of traditional visual search tasks, as used in Pambakian et al., 2004. and Microsoft Kinect as part of physical therapy (e.g. [9][10]). In addition, many bespoke game-based tools have been developed to augment physical therapy (i.e. [3]) and mental health therapy (see [15]) with promising results.

\section{Rehabilitation for Visual Impairment}

This project focuses on designing game based therapy for the rehabilitation of functional vision for children with hemianopia and childhood cerebral visual impairment. Both hemianopia and CVI are visual impairments that are caused by damage or disease in areas of the brain involved with visual processing. Hemianopia is a loss of one half of a person's vision caused by damage to one side of the visual processing areas of the brain. A diagnosis of CVI is often given when there is a more complex loss of visual perception (or multiple losses) such as the inability to see an object when it is placed on a complicated background or next to other objects. People with these visual impairments can have difficulties with mobility (e.g. crossing the road) and close work (e.g. reading or finding an object on a table).

Evidence has accumulated that rehabilitation strategies for patients with neurological visual impairment are effective. The current project focuses on the use of compensatory strategies (see [8]). Specifically, eye and head movement strategies can be learned to improve the effectiveness of visual scanning. This learned scanning allows sufferers to perceive parts of the visual field that would otherwise be inaccessible (i.e., people with left visual field loss will learn to compensate by making large and frequent eye movements to the left so that the intact visual field on the right can see what would otherwise be out of sight). Compensatory scanning allows adults with visual field loss to function more effectively in the activities of daily life. Therapy requires months of training to be effective, but as the new strategy becomes habitual patients can focus attention on the activity of interest rather than on maintaining the compensatory eye movements [13].

Compensatory eye movement strategies can be learned through using simple visual search tasks (see fig 1 . for example tasks) [13]. These tasks involve the presentation of an array of monochrome 2D shapes on a black background. Patients are required to search the array of stimuli to find a target or "odd-one-out". Interestingly, there is no interaction required of the patient other than visual searching. They are not required to indicate when they have found the target, nor is there any checking by the system whether they have scanned the visual field effectively. A typica session involves the presentation of hundreds of these search tasks within a 30 minute window and sessions must be carried out daily for a number of months in order to see improvements in functional vision.

While visual search tools can teach effective scanning techniques, they are repetitive and uninteresting, and, we suggest, unsuitable for use with children. Indeed, similar tasks, which are used for the formal assessment of visual fields have been criticised as unsuitable for use with children, due to their poor engaging qualities [1]. We are interested in exploring how the engaging qualities of the visual search training can be improved through the design of therapy tools based on game design principles. To our knowledge, no tools currently exist for the visual rehabilitation of children with CVI, and certainly none that are designed to maximise engagement and adherence. 


\section{Design Requirements}

\section{Game Design}

The definition of what constitutes an engaging game has been discussed at length (see [14]) but a useful summary is provided by Linehan et al., [11]: 1) games typically present the player with a series of short, medium and long-term goals, 2) they typically require the player to take some actions or decisions in order to reach those goals, 3) they excel at providing

immediate, appropriate and specific feedback to players, 4) they often have a complex system for presenting players with rewards for achievement, 5) long, complex tasks are broken down into short, simple components, which are trained individually before being chained together. 6) players are expected to demonstrate excellent performance of a skill before they can advance to using that skill in a more challenging environment, 7) Where games present the player with options for taking action, no one action should be obviously correct, while others are obviously incorrect. Of course, this list is in no way exhaustive.

\section{Games for Therapy}

There are a number of overarching concerns for the design of useful therapeutic games. Firstly, therapyappropriate behaviour must form the basis of the game mechanics [3]. For example, if the therapy is designed to improve dynamic balance (i.e., [9][10]), the principal way in which players interact with, achieve success in, and progress through, the game should be through measures of their ability to balance. The game should not require difficult balance activities interspersed with game-like fun activities. This concept is similar to that of "intrinsic learning" [5] seen in the design of educational games.

Secondly, due to the typically wide range of abilities observed with users of therapeutic games, it is essential to allow adaptability based on patient ability [4][3][15][1]. For example, through the process of developing game-based stroke rehabilitation technology for elderly patients, Flores et al., [3] found that it was important to allow the user, carer, or therapist (as appropriate) the ability to adapt complexity of both the visual content and the cognitive challenges presented by the game.

Thirdly, social interaction is one of the strongest predictors of the amount of time people spend playing games (e.g., [7]). Since those undergoing therapy will also commonly need to be supervised or supported by therapists or carers, it seems appropriate to include social interaction (with carers, parents, siblings or friends) as part of the game design (see [6]). However, in order to achieve this goal, the game design must provide engaging challenges for players of all abilities [12]. Finally, the game should allow the therapist, carer or patient to track performance over time, in order to allow for intuitive judgments over whether progress is being achieved [1]. 

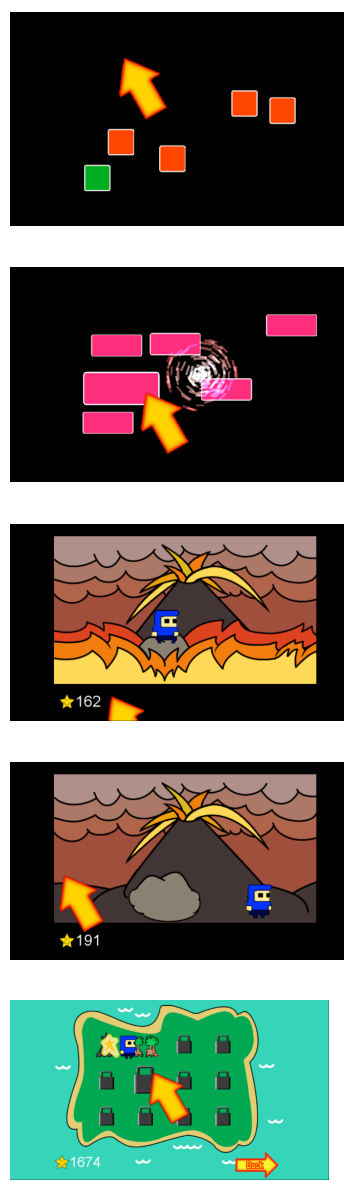

Figure 2. Five screenshots from the prototype game. Panels (a) and (b) demonstrate search tasks, while (c)(e) show screens that signal progression.

\section{Specific requirements}

While visual search based therapy is effective, we have no evidence to explain which features of those search tasks are essential, and which are not. Thus, it seems necessary to replicate the physical and behavioural characteristics of traditional visual search tasks. The core mechanic of the game should involve the repetitive visual searching of an array of distractor stimuli in order to find a target. In addition, since players are visually impaired, it is important to keep the visual aspects of the game simple and to augment them with other forms of representation, such as audio.

\section{Design}

Best practice in game design and development specifies rapid iteration, where real players are placed at the heart of the process and all development is evaluated in a formative manner [14]. Therapeutic games (i.e., [6][4]), add the complexity that games must be evaluated not only for their ability to engage and entertain, but also to fulfill their therapeutic goals. During development, data will be collected in an iterative, formative manner through semi structured interviews with therapists, parents, carers and the patients themselves, as well as through quantitative analysis of performance of players on the game tasks.

The goal of iterative development in the design of therapeutic games should be to measure whether individual game elements elicit the types of behaviour identified as necessary for successful therapy. An analogy can be drawn with the development of psychometric instruments. In both cases, work is carried out in an iterative manner to evaluate whether specific instances represent a valid test of a wider concept or skill. Formative assessment must focus clearly on evaluating whether or not game design elements promote compensatory visual searching strategies, and prolonged engagement, which are the behaviours identified in our project as necessary for successful therapy.

We have implemented a game prototype, the core mechanic of which requires players to visually search arrays of coloured shapes in order to find specified targets, and to click on those targets. Requiring players to click introduces interactivity, which is absent from the traditional search tasks, and allows for the delivery of feedback based on performance. Correct clicks trigger both audio effects, and occasionally visual effects. We have also included long term (escape from an island), and medium term, (overcome obstacles on your path) goals to add meaning and a sense of progression to the search tasks. Screenshots of the game can be seen in Figure 2 .

\section{Early Findings}

Four participants, aged 15-18, engaged in separate fortnightly meetings with researchers over a three month period. Meetings were held in a familiar room at their school and lasted for approximately thirty minutes. During sessions, participants were observed while play-testing the game and asked to make comments on the game visuals, controls and mechanics. Two of these learners have participated in a single play-test of a two player prototype of the game. Additionally, one-off meetings have taken place with a group of three young people with acquired brain injury that were attending a local occupational therapy training scheme, and with a group of eight therapists who work with visually impaired young people on a daily basis. 
Data Collection. The intention was to audio record play sessions. Immediately, we found an obstacle to this strategy, as many participants, particularly those with verbal dyspraxia (an impairment of the fine articulatory motor skills required to produce clear speech) did not consent to audio recording. Thus, initial data primarily takes the form of notes taken by the researcher.

Perceptual. Players with CVI can often have difficulty perceiving complex visual images. In contrast players with hemianopia (and no other CVI symptoms) will often still have good vision in their intact visual field. Although we were keen to design features that would work equally well across a range of abilities it was clear that if objects were too small or too closely packed together it became impossible for participants to discriminate between them. We have implemented an options menu that can set the level of a small number of fundamental visual properties (e.g. size and number of shapes in an array) depending on the specific visual ability of each player, with encouraging early results.

Cognitive. As some of our players have impaired cognition we wanted to be certain that the game mechanics would be easy to understand. Feedback for short term goals is delivered as soon as the player selects a shape in the visual array. Originally, regardless of whether the response was correct or incorrect, the game would continue to the next stage. To make the cause and effect of rewards and behaviour more clear, we implemented a different reward system in which players must find the correct target shape before they can progress. This solution encouraged participants to visually search the screen when they selected a shape and nothing happened.
Motor. Initially we were concerned that using a mouse to control the game would require a degree of manual dexterity that some players would find difficult.

However, given the choice between the mouse and pressing buttons on the keyboard all our participants chose to use the mouse, stating that they found the mouse a more intuitive controller, not necessarily because the mouse is easier to control. Participants have also expressed an interest in playing the game using a touchscreen or tablet device, which we are currently exploring. Additionally for those players with severe impairment of motor coordination (e.g. players with associated cerebral palsy or hemiplegia) we have investigated the possibility of using access technology such as large button boxes and eye tracking devices.

Social. We have implemented a 2-player, turn-taking version of the game. Initial findings from two participants are promising in terms of player engagement. Both players preferred playing together than on their own. However, a concern arose from a therapy point of view. Specifically, sharing a screen required both players to sit either side of the centre of the screen. This is problematic as it changes the participants field of view relative to the items presented on-screen, and therefore impacts their visual search behaviour. We intend to address this issue in the next iteration.

\section{Discussion}

We have outlined requirements for the design and development of a game for the rehabilitation of functional vision for children with cerebral visual impairment. No tools exist for the rehabilitation of children with CVI, and certainly none that are designed to maximise engagement. We are undertaking the 
iterative design, development and evaluation of the game, in co-operation with a specialist centre for childhood visual impairment. Early work identifies a number of areas that must be improved. Longer term, we aim to carry out summative evaluation of the game through a controlled study, in which we carry out pre and post evaluation of functional vision.

\section{Acknowledgements}

This work was supported by The WESC Foundation, the Technology Strategy Board, and the Medical Research Council of the United Kingdom, as part of a Knowledge Transfer Partnership (Ref: KTP008989).

\section{References}

[1] Allen, L. E., Slater, M. E., Proffitt, R. V., Quarton, E., \& Pelah, A. A new perimeter using the preferential looking response to assess peripheral visual fields in young and developmentally delayed children. J. Am. Assoc. Pediatric Ophthalmology and Strabismus, 16 (2012), 261-265.

[2] Annema, J. H., Verstraete, M., Abeele, V. V., Desmet, S., \& Geerts, D. Video games in therapy: a therapist's perspective. International Journal of Arts and Technology, 6 (2013), 106-122.

[3] Flores, E., Tobon, G., Cavallaro, E., Cavallaro, F. I., Perry, J. C., \& Keller, T. Improving patient motivation in game development for motor deficit rehabilitation. In Proc ACE 2008, ACM Press (2008) 381-384.

[4] Goh, D. H., Ang, R. P., \& Tan, H. C. Strategies for designing effective psychotherapeutic gaming interventions for children and adolescents. Computers in Human Behavior, 24 (2008), 2217-2235.

[5] Habgood, M. J., \& Ainsworth, S. E. Motivating children to learn effectively: Exploring the value of intrinsic integration in educational games. The Journal of the Learning Sciences, 20 (2011), 169-206.
[6] Hernandez, H. A., Ye, Z., Graham, T. C., Fehlings, D., \& Switzer, L. Designing action-based exergames for children with cerebral palsy. In Proc CHI 2013, ACM Press (2013), 1261-1270.

[7] Jansz J, Martens L. Gaming at a LAN event: the social context of playing video games. New Media Soc, 7 (2005), 333-355.

[8] Kerkhoff, G. Neurovisual rehabilitation: recent developments and future directions. J. Neurology, Neurosurgery \& Psychiatry, 68 (2000), 691-706.

[9] Lange, B., Flynn, S., Proffitt, R., Chang, C. Y., \& Rizzo, A. S. Development of an interactive game-based rehabilitation tool for dynamic balance training. Topics in Stroke Rehabilitation, 17 (2010), 345-352.

[10] Lange, B., Chang, C. Y., Suma, E., Newman, B., Rizzo, A. S., \& Bolas, M. Development and evaluation of low cost game-based balance rehabilitation tool using the Microsoft Kinect sensor. In Proceedings EMBC 2011, IEEE (2011), 1831-1834.

[11] Linehan, C., Kirman, B., Lawson, S., \& Chan, G. (2011, May). Practical, appropriate, empiricallyvalidated guidelines for designing educational games. In Proc CHI 2011, ACM Press (2011), 1979-1988.

[12] Ossmann, R., Miesenberger, K., \& Archambault, D. (2008). A computer game designed for all. In Computers Helping People with Special Needs, Springer (2008), 585-592.

[13] Pambakian, A. L. M., Mannan, S. K., Hodgson, T. L., \& Kennard, C. Saccadic visual search training: a treatment for patients with homonymous hemianopia. Journal of Neurology, Neurosurgery \& Psychiatry, 75 (2004), 1443-1448.

[14] Salen, K., \& Zimmerman, E. Rules of Play: Fundamentals of Game Design. MIT Press, 2003.

[15] Wilkinson, N., Ang, R. P., \& Goh, D. H. Online video game therapy for mental health concerns: $A$ review. International journal of social psychiatry, 54 (2008), 370-382. 
